\title{
High Resolution EELS of Point Defects in a Nitride Semiconductor Material
}

\author{
Katia March ${ }^{1}$, Shuo Wang ${ }^{2}$, Fernando A. Ponce ${ }^{2}$ and Peter Rez ${ }^{2}$ \\ 1. Eyring Materials Center, OKED, Arizona State University, Tempe, AZ. \\ 2. Department of Physics, Arizona State University, Tempe, AZ.
}

There is currently considerable interest in B doping of $\mathrm{AlN}$ and GaN semiconductors to engineer the band gap for UV laser applications.

We have studied a BA1N thin film grown by MOCVD with gas-flow $\mathrm{B} /(\mathrm{B}+\mathrm{Al})$ ratio of $18 \%$. The boron content was measured to be $\sim 16 \%$ by Rutherford backscattering, but only $9 \%$ by X-ray diffraction. The discrepancy has been explained as B segregation at twin boundaries [1]. However, it's also possible that some of boron atoms are on interstitial sites, instead of replacing Al in the film.

Fig 1 shows a high resolution HAADF image of the BAlN film grown on the AlN substrate. It was recorded at $100 \mathrm{kV}$ in the NION HERMES ${ }^{\text {тм }} 100$ with a beam convergence of $30 \mathrm{mrad}$ using the aberration corrector. The position of the atomic columns is well defined in the AIN, but less clear in the BAIN, probably due to defects. The darker regions have higher B concentration, which correlated with maps of intensity in the B K edge.

Transitions involving energy levels associated with point defects are often associated with optical or IR emission, less than $2 \mathrm{eV}$. These can be resolved with the use of the monochromator in the NION HERMES $^{\text {тм }} 100$, this time operated at $60 \mathrm{kV}$. A lower accelerating voltage is needed to avoid Cerenkov losses, which would give a background in the bandgap region. Fig 2a shows features with energies ranging from 0.2 to $0.8 \mathrm{eV}$, recorded by scanning the $11 \mathrm{~nm} \times 11 \mathrm{~nm}$ region shown in Fig $2 \mathrm{~b}$. They show a threshold followed by a gradual decrease, similar to that seen for core edges. This is to be expected, as it is possible to excite to higher energy states in the conduction band.

To identify which defects might be responsible for these features, VASP DFT calculations were performed using PAW LDA potentials for single $\mathrm{Al}, \mathrm{B}$ and $\mathrm{N}$ interstitial atoms, and single $\mathrm{Al}$ and $\mathrm{N}$ vacancies, in a supercell constructed from $3 \times 3 \times 3$ AlN unit cells. The structures were initially relaxed with $3 \times 3 \times 3 \mathrm{k}$ points. Subsequently a high-resolution density of states (DOS) was calculated with $5 \times 5 \times 5$ $\mathrm{k}$ points. The bandgap for AIN from the DOS shown in Fig 3a is underestimated as $4.6 \mathrm{eV}$, as is typical for DFT. The calculations also determined the position of the Fermi level shown as $0.0 \mathrm{eV}$, so plausible transitions to the empty states in the conduction band could be identified. Only the B and Al interstitials as shown in Figs $3 b$ and Fig 3c, and $\mathrm{N}$ vacancies, showed levels that matched those shown in Fig $2 \mathrm{a}$. Differences between measured energy levels and the DFT results can be attributed to limitations in DFT.

Detection of point defects is the first step in mapping their location at $\mathrm{nm}$ resolution, once the functional form of the point spread function is known [2].

\section{References:}

[1] S. Wang et al, J. Cryst. Growth 475 (2017), p. 334.

[2] The use of facilities within the Eyring Materials Center at Arizona State University is acknowledged. 
X. Li, T. Detchprohm and R.D. Dupuis are acknowledged for growing the materials.

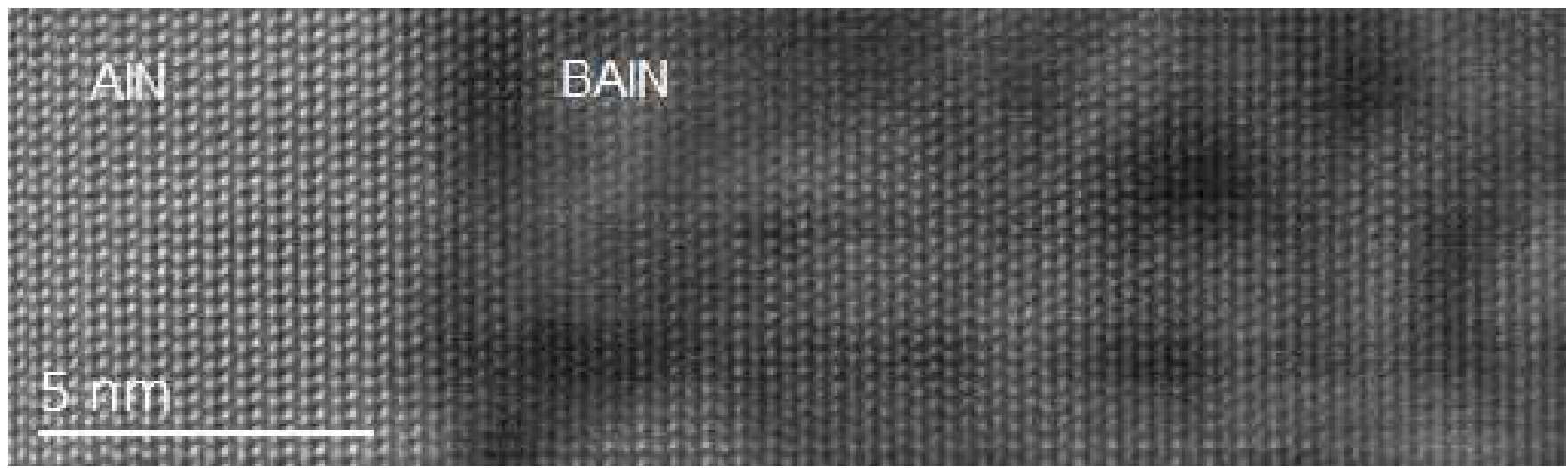

Figure 1. HAADF image showing AlN and B implanted region.

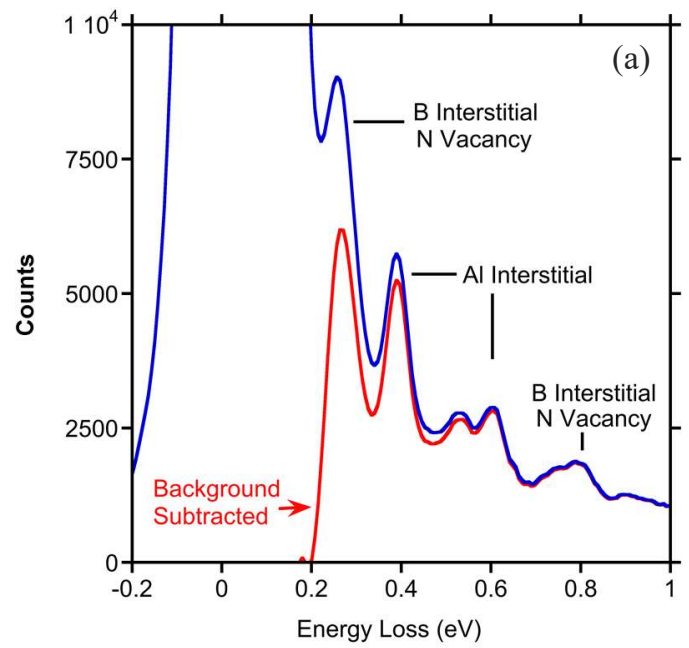

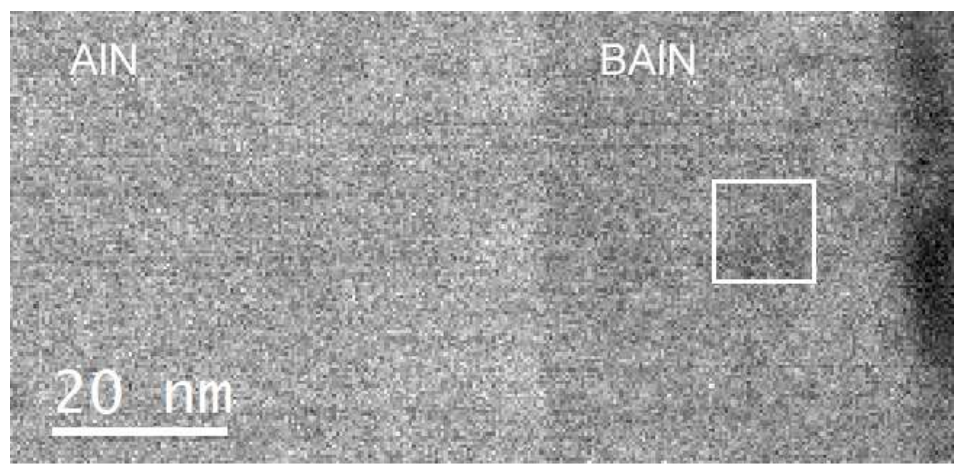

(b)

Figure 2. (a) Spectrum features arising from point defects (b) Region from which spectrum was taken.

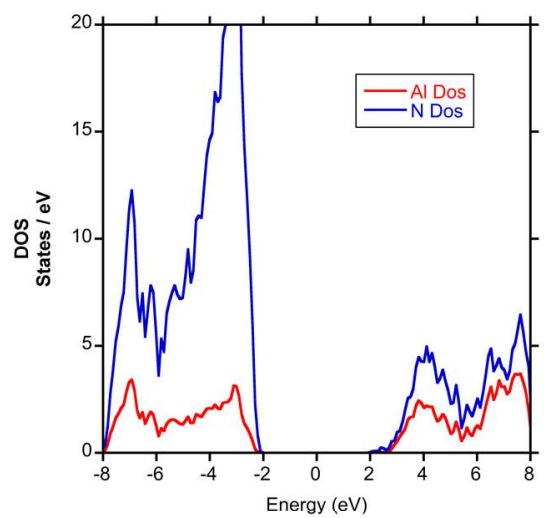

(a)

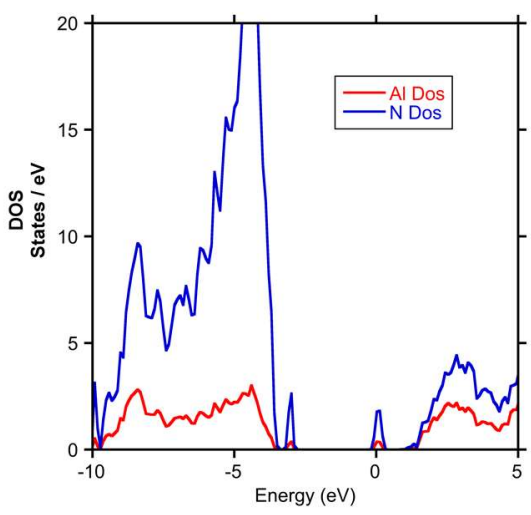

(b)

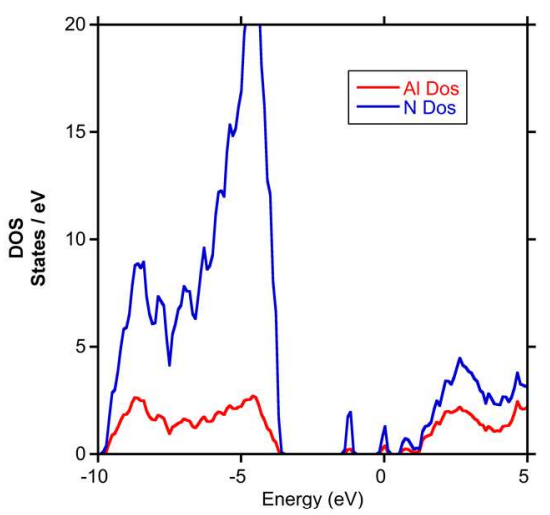

(c)

Figure 3. DFT calculations for (a) AlN, (b) with B and (c) with Al interstitial in $3 \times 3 \times 3$ supercell. 